\title{
Educación transdisciplinar en valores para la integración de jóvenes en riesgo de exclusión social
}

\author{
Educação transdisciplinar em valores na integraçao de jovens em \\ risco de exclusão social
}

\section{Value transdisciplinary education in the integration of young people with social exclusion risk}

\author{
Anfrisia Alessandra Pereira Araujo'
}

IES Montes Orientales, Iznalloz, Granada, España, Colaboradora de Educación Social

\section{María del Carmen Plata Luque ${ }^{2}$}

IES Montes Orientales, Iznalloz, Granada, España, Profesora del Departamento de Biología

\section{María Luisa Ortiz Mallol ${ }^{3}$}

IES Montes Orientales, Iznalloz, Granada, España, Profesora del Departamento de Historia y Geografía

\section{Ana Isabel Jiménez Durán ${ }^{4}$}

IES Montes Orientales, Iznalloz, Granada, España, Profesora del Departamento de Música

Resumen: El artículo describe la relevancia de la integración de jóvenes de los grupos étnicos gitano y no gitano, en riesgo de exclusión social, utilizando como núcleo central los valores humanos y la transdisciplinaridad. El principal objetivo es la inclusión de la educación en valores en la educación conceptual, intelectual, emocional y práctica del curriculum, de modo que la importancia de los valores trascienda al contexto familiar, social y cultural. A partir de referentes teóricos de la educación, la sociología y la teoría de sistemas (Freire, Bordieu, Morin, Nicolescu) la metodología parte de los modelos que integran paradigmas objetivistas, subjetivistas y crítico. El principal resultado de este enfoque es una formación y una capacitación sistemática en valores en el ámbito de una educación formal inclusiva y participativa. Palabras clave: Educación en Valores. Transdisciplinaridad. Integración Social. Educación Inclusiva y Participativa.

Máster y Graduada en Educación Social por la Universidad de Granada; https://orcid.org/0000-0002-3025-7275; http://lattes. cnpq.br/6071682846491756.

2 Doctora y Licenciada en Biología por la Universidad de Córdoba; https://orcid.org/0000-0003-0121-3713; http://lattes.cnpq. $\mathrm{br} / 7823431375159277$.

3 Licenciada en Historia y Geografía por la Universidad de Granada; https://orcid.org/0000-0002-7904-0536; http://lattes.cnpq. $\mathrm{br} / 5132583221872050$.

4 Licenciada en Historia por la Universidad de Granada; https://orcid.org/0000-0002-9277- http://lattes.cnpq. br/5113024139669363. 
Resumo: 0 artigo descreve a relevancia da integraçao de jovens dos grupos étnicos cigano e não cigano, em risco de exclusão social, utilizando como núcleo central os valores humanos e a transdisciplinariedade. 0 objetivo é a inclusão da educação em valores na educação conceptual, intelectual, emocional e prática das diferentes disciplinas do curriculum, que possa permear ao contexto familiar, social e cultural a importância dos valores humanos. Partindo de referenciais teóricos da educação, da sociologia e da teoria de sistemas (Freire, Bordieu, Morin, Nicolescu) a metodologia tem como base os modelos que integram paradigmas objetivistas, subjetivistas e críticos. 0 principal resultado desta perspectiva é a formação e capacitação sistemâtica em valores no âmbito de uma educação formal inclusiva e participativa.

Palavras-chave: Educação em Valores. Transdisciplinaridade. Integração Social. Educação Inclusiva e Participativa.

Abstract: This article describes how relevant the human values and transdisciplinarity concepts are in the integration of two ethnic groups (gypsy and caucasian), both in risk of social exclusion. The main objective is the inclusion of the value education inside the conceptual, theoretical, emotional and practical formal education with the aim of translating this values to the family, social and cultural environment. Using theoretical references from education, sociology and system theory (Freire, Bordieu, Morin, Nicolescul the methodology starts from models which integrate objective, subjective and critical paradigms. The main result of this approach is an education in values in the context of the formal education which is inclusive and participatory.

Keywords: Human values. Transdisciplinarity. Social Integration. Inclusive and Participatory Education.

Recibido el 4 de julio de 2018 Aceptado el 13 de febrero de 2019

Publicado el 25 de marzo de 2019

\section{INTRODUCCIÓN}

La enseñanza formal tradicional basada en el aprendizaje de contenidos especializados y separados en diferentes materias de estudio se ha mostrado útil durante décadas. Sin embargo, en el mundo global en el que vivimos es necesario adoptar un enfoque integrador. A ello se suma, como ponemos de manifiesto en este artículo, que en entornos con graves problemas sociales, económicos y educativos los estudiantes tienden a distanciarse de estas formas educativas debido a múltiples factores.

En los últimos tres años hemos trabajado en uno de estos entornos con graves dificultades socio-económicas y culturales y con una población donde la etnia gitana es muy 
numerosa. Mediante un estudio diagnóstico de intervención socioeducativa previo a este proyecto (PEREIRA, 2016) hemos detectado que la educación en valores es necesaria para fomentar la integración de las diferentes etnias que conviven en este entorno. Además de ello, no es suficiente con una formación específica en valores, sino que se hace necesario incluir esta formación en las disciplinas tradicionales. En este sentido, la integración de las diferentes disciplinas curriculares con un enfoque transdisciplinar, en el que se entrelaza la formación tradicional con una perspectiva de los valores humanos, se ha mostrado muy interesante para atraer el interés de los educandos y para fomentar un proceso de integración educativa, cultural y social en un entorno tan complejo.

Hemos realizado nuestro trabajo mediante un proyecto participativo y colaborativo al que denominamos Proyecto Transdisciplinar en Valores (en adelante, PTV). El proyecto se ha desarrollado en un Instituto de Educación Secundaria de una de las zonas más deprimidas de España. En él han participado un gran número de profesores del centro y los estudiantes del primer curso de la educación secundaria.

Esta experiencia nos ha demostrado que la educación en valores humanos, mediante un enfoque transdisciplinar, es muy interesante para abordar la integración de jóvenes de diferentes grupos étnicos en riesgo de exclusión social. En primer lugar, presentaremos un diagnóstico socio-educativo para comprender las dificultades y los retos con los que se enfrenta el sistema educativo en un entorno tan diverso. A continuación expondremos un breve resumen del marco teórico que nos ha permitido definir el proyecto. Seguiremos con el marco legislativo español en el que se ha desarrollado y que da cabida a este tipo de iniciativas. Sin embago, no basta con disponer de un marco teórico y el salto a la práctica resulta difícil, en el siguiente apartado presentaremos el marco estratégico que nos ha permitido implementar el proyecto.

Por último, expondremos los detalles del proyecto y la evaluación de sus resultados a partir de los cuales podemos considerar la transdisciplinaridad y la educación en valores como un enfoque útil e interesante en estos entornos.

\section{CONTEXTUALIZACIÓN DE LA ZONA DE ESTUDIO}

El ámbito en que se desarrolla el PTV es el Instituto de Educación Secundaria "Montes Orientales", que está ubicado en el pueblo de Iznalloz a 36 km de Granada capital, en el sur de España. Además, está situado en la comarca de los Montes Orientales de Granada, una zona especialmente deprimida económicamente con serios problemas de absentismo escolar. 


\subsection{ESTRUCTURA DEMOGRÁFICA ECONÓMICA Y SOCIAL}

Esta comarca tiene una población de 29.530 habitantes con una densidad de 19,32 hab/kma (INSTITUTO NACIONAL DE ESTADÍSTICA, 2017). Estos datos indican la gran dispersión de la población en una localidad agraria con una significativa parte de su superficie ocupada por montes y olivos.

Iznalloz tiene 5.033 habitantes, de los cuales más de $40 \%$ son población gitana, siendo menores de 25 años aproximadamente el $40 \%$ y presentando los peores índices económicos de Granada y una da las rentas per cápita más baja de la Unión Europea (INSTITUTO NACIONAL DE ESTADÍSTICA, 2017).

Mapa 1 - Evolución de la población

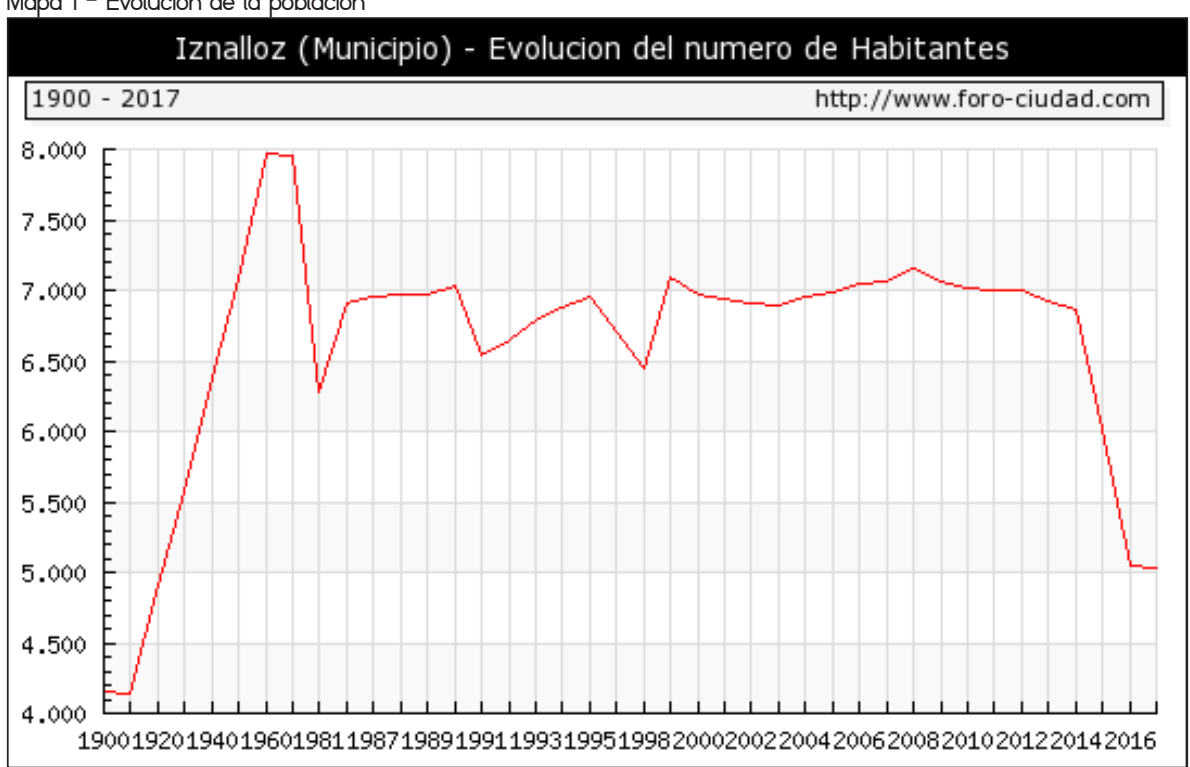

Fuente: Foro Ciudad (2017).

En la actualidad hay 2.569 hombres y 2.464 mujeres, con una notable disminución poblacional en los últimos años (Mapa 1). En la pirámide poblacional predominan menores de 60 años y, en especial, hay un incremento de la población infantil y juvenil.

Los indicadores socioeconómicos de esta localidad son los propios de las áreas de montaña interiores, en la que se podría indicar que los procesos económicos se encuentran en una situación importante de lentitud, principalmente por carencias demográficas, culturales y formativas. 
La economía de esta localidad se basa principalmente en la agricultura, ganadería y sus derivados, seguida, con un déficit importante en los últimos años, del sector de la construcción, el comercio y talleres. El trabajo es temporero en la recogida de la aceituna desde diciembre a febrero, después hay una larga temporada de paro hasta que se inician las campañas de frutas y hortalizas en otras regiones de España y en Francia. Produciéndose una emigración temporal masiva de marzo a junio y en agosto y septiembre. Es importante destacar que los sectores menos representativos son los de las áreas sanitarias, servicios sociales e industrias extractiva y manufacturera.

En general, los indicadores socio-económicos de esta comarca muestran una situación económica frágil y deprimida, muy dependiente de una agricultura que se encuentra fuertemente subvencionada. A título de ejemplo baste mencionar que casi el $20 \%$ de la población activa recibe subsidios gubernamentales (SISTEMA..., 2017).

La alta tasa de desempleo unida a una alta temporalidad de la comarca de los Montes ha registrado históricamente un desempleo endémico que ha llegado a superar el $40 \%$ en los duros años de la crisis (2012 a 2018), aumentando aun más en el caso de las mujeres hasta alcanzar un $57 \%$ de desempleo. En la actualidad encontramos un descenso de estos índices hasta obtener una media en toda la comarca del $16,6 \%$ de paro en 2017 , siendo algo más elevado en Iznalloz con un 17,93\%. Predominan los contratos temporales (5.893) frente a los contratos indefinido (43) (SISTEMA..., 2017).

\subsection{DIAGNÓSTICO EDUCATIUO-SOCIAL}

El resultado socioeconómico y sociocultural de esta compleja realidad incide en los centros escolares del municipio de manera negativa, destacando el absentismo estructural, el abandono temprano del sistema educativo, falta de interés de las familias por el desarrollo escolar de sus hijos, falta de expectativas personales y de mejora, familias desestructuradas, abandono y maltrato infantil. En 2013 el servicio social de Iznalloz informa haber llevado en adopción a dieciséis niños de la etnia gitana por maltrato infantil.

La consecuencia de esta situación es la existencia de una mayor probabilidad de riesgo y exclusión social, reflejado en actitudes delictivas y de marginalidad, que influyen en los centros educativos en aspectos como la puntualidad, las calificaciones académicas, cuidado del material, actitud ante el trabajo, el respeto hacia las personas, la responsabilidad y la valoración positiva del sistema educativo público.

Es evidente que la situación del absentismo estructural, del abandono escolar, del maltrato infantil se produce en todos los estratos de la sociedad, independientemente de 
la clase social en la que se encuentre el sujeto. Sin embargo, es importante destacar que la clase social desfavorecida es la más perjudicada.

No obstante, es importante resaltar que después de muchos años de trabajo se detecta, en el ámbito general, que los gitanos han pasado de ser en su mayoría analfabetos a tener la totalidad de sus hijos escolarizados y aumentar el número de jóvenes que continúan en los estudios secundarios obligatorios (ESO). Este resultado es debido principalmente al aumento de confianza de las familias gitanas en el sistema educativo público, así como al aumento de las facilidades para el acceso al mismo (LIÉGEOIS, 1998).

Debemos destacar que una de las características fundamentales del sistema educativo público español es su notable crecimiento en los últimos 20 años, tanto en el ámbito cuantitativo como cualitativo. Actualmente se puede decir que, al igual que el resto de los países de la Unión Europea, España ha alcanzado la denominada universalización de la educación para todos los niños y jóvenes, sin distinción de sexo, entre la edad de 6 a 16 años. Además, muchos jóvenes de 17 y 18 años se insertan en enseñanzas postobligatorias. No obstante, el número de analfabetos funcionales o personas sin estudios y los niveles de absentismo y abandono escolar continúan siendo una de las mayores debilidades existentes en España.

Todos estos condicionantes socio-económicos se manifiestan en las actitudes hacia el sistema educativo y el nivel de compromiso de las familias en la educación de sus hijos. En el Cuadro 1 hemos resumido los principales elementos diagnósticos que han sido detectados en el entorno social y educativo y que conforman la realidad con la que trabajamos.

\subsection{CONTEXTUALIZACIÓN DE LA COMUNIDAD EDUCATIUA}

El Instituto de Educación Secundaria Obligatoria "Montes Orientales" está situado en el municipio de Iznalloz y, además de ofrecer la enseñanza de la ESO (Enseñanza Secundaria Obligatoria), es el único que oferta los estudios de Bachillerato y Formación Profesional en toda la comarca de los Montes Orientales.

El centro cuenta con 71 profesores para una población de 750 educandos. Entre estos hemos seleccionado a los 104 alumnos de $1^{\circ}$ de ESO, que tienen una edad media de 12 años y cuyos estudios se corresponden con la sexta/séptima serie de la educación primaria en Brasil. Dentro de este grupo están incluidos educandos de Compensación Educativa (15 alumnos, mayoritariamente de etnia gitana) y educación especial (7 educandos), algunos de los cuales llegan a los 14 años. 
Cuadro 1 - Elementos diagnósticos socio-educativos

\begin{tabular}{|c|c|c|}
\hline & \multicolumn{2}{|c|}{ Ambas etnias } \\
\hline \multirow[t]{2}{*}{$\begin{array}{l}\text { Aspectos externos } \\
\text { (socioculturales, económi- } \\
\text { cos, axiológicos) }\end{array}$} & \multicolumn{2}{|c|}{$\begin{array}{l}\text { - Buena parte de los ingresos dependen de subvenciones o ayudas. Fuerte } \\
\text { dependencia económica de subsidios del gobierno. } \\
\text { - No existen centros culturales en el pueblo. } \\
\text { - Etnocentrismo local. } \\
\text { - No cuidan la ciudad, no reciclan la basura. } \\
\text { - Inercia política: el ayuntamiento no se involucra en el desarrollo del pueblo. } \\
\text { - Malas condiciones educativas y de maduración de hijas y hijos. }\end{array}$} \\
\hline & Población gitana & Población no gitana \\
\hline $\begin{array}{l}\text { Aspectos externos } \\
\text { (socioculturales, económi- } \\
\text { cos, axiológicos) }\end{array}$ & $\begin{array}{l}\text { - Desinterés por la educación, formaci- } \\
\text { ón y por las actividades culturales. } \\
\text { - Consumo y tráfico de drogas. } \\
\text {-Absentismo y fracaso escolar. Menos } \\
\text { de } \% \text { llegan a bachillerato. } \\
\text { - 90\% no continuarán con los estudios } \\
\text { después de la ESO. } \\
\text { - Buenas habilidades artísticas (baile/ } \\
\text { cante flamenco). } \\
\text { - Organización social basada en } \\
\text { el parentesco (patrilineal) y familias } \\
\text { numerosas. } \\
\text { - Religión evangélica dominante. } \\
\text { - Casas inadecuadas en muchos } \\
\text { casos. } \\
\text { - Concentración (vivienda) en determi- } \\
\text { nados barrios, dificultando el proceso } \\
\text { de integración. } \\
\text { - Dificultad de comunicación directa con } \\
\text { las familias. } \\
\text { - Se dedican a la venta ambulante. } \\
\text { - Renta económica menor de } 6.000 / \\
\text { año. } \\
\text { Estatus socioeconómico y educativo } \\
\text { bajo. } \\
\text { - Emigración estacional por trabajo, }\end{array}$ & $\begin{array}{l}\text { - Mayor interés por el estudio. } \\
\text { - Desprecio por la cultura gitana. } \\
\text { - Consumo y tráfico de drogas. } \\
\text { - Abandono escolar después de los } 16 \\
\text { años (30\%). } \\
\text { - Menor fracaso escolar, concluyen la } \\
\text { ESO el } 70 \% \\
\text { - No tienen tanta afinidad con el arte. } \\
\text { - Número de hijos entre } 1 \text { a } 3 . \\
\text { - Religión católica. } \\
\text { - Hábitat más estructurado. Viven } \\
\text { apartados de los gitanos (dificultad de } \\
\text { integración). } \\
\text { - Se puede contactar con estas } \\
\text { familias. } \\
\text { - Tienen negocios propios. } \\
\text { - Renta económica entre } 12.000 \text { y } \\
\text { Estatus socioeconómico y educativo } \\
\text { más elevado. } \\
\text { - Los jóvenes con estudios empiezan } \\
\text { a abandonar el pueblo. }\end{array}$ \\
\hline
\end{tabular}




\begin{tabular}{|c|c|c|}
\hline $\begin{array}{l}\text { Aspectos internos } \\
\text { (cognitivos, emocionales, } \\
\text { comportamentales) }\end{array}$ & $\begin{array}{l}\text { - No existe interés en asimilar las } \\
\text { materias de estudio. } \\
\text { - No dedican tiempo para estudiar en } \\
\text { casa. } \\
\text { - Capacidades intelectuales no } \\
\text { desarrolladas. Poca motivación para el } \\
\text { aprendizaje. } \\
\text { - Poca motivación para desarrollar las } \\
\text { aptitudes artísticas. } \\
\text { - Existe una segregación con los no } \\
\text { gitanos. } \\
\text { - Viven más influidos por la cultura de } \\
\text { la marginación que por la cultura gita- } \\
\text { na. Están perdiendo las costumbres. } \\
\text { - Autoconcepto más negativo. } \\
\text { - Autoestima baja. } \\
\text { - Menor empatía. } \\
\text { - La mentira una actitud añadida a sus } \\
\text { prácticas. }\end{array}$ & $\begin{array}{l}\text { - Asimilan las materias de estudios } \\
\text { fundamentalmente para aprobar el } \\
\text { curso. } \\
\text { - Tienen más hábito de estudio. } \\
\text { - Capacidades artísticas poco desar- } \\
\text { rolladas. } \\
\text { - No identifican manifestaciones cultu- } \\
\text { rales propias. } \\
\text { - Viven separados de los gitanos. } \\
\text { - No aprendieron apreciar la cultura } \\
\text { gitana } \\
\text { - Amistad entre no gitanos. } \\
\text { - Autoconcepto más positivo. } \\
\text { - Autoestima más positiva. } \\
\text { - Algo de empatía. } \\
\text { - Empiezan a desarrollar la mentira } \\
\text { como un mecanismo de logros. }\end{array}$ \\
\hline
\end{tabular}

Fuente: los autores.

Nos hemos decidido por estos educandos ya que al comenzar sus estudios de ESO podrán integrarse y sensibilizarse con temas relacionados con la vida y con los valores. Además, ello permite que estos mismos educandos continúen obteniendo esta formación hasta el final de la educación secundaria.

\section{MARCO TEÓRICO: LA TRANSDISCIPLINARIDAD Y LOS UALORES}

En el mundo globalizado en que vivimos la exclusión social supone una situación de partida muy complicada y, a la vez, compleja. Según Bendit y Hahn-Bleibtreu (2008), muchas personas viven la transición a la vida activa como una etapa doblemente compleja al tener que enfrentarse, desde una situación carencial, a una serie de barreras muy diversas que obstaculizan su participación en la sociedad y que atraen problemas de convivencia, exclusión y marginación social. Ante este doloroso escenario el resultado se ve reflejado en actitudes desctructivas, envueltas en rechazo y alienación, que según Bordieu (2007) son manifestaciones concretas de una "violencia simbólica" extendida, que afecta y domina las relaciones sociales y, a la vez, genera conflictos afectivos, emocionales, psicológicos, sociales, etc., trayendo con ellos unos resultados demoledores. 
Como consecuencia de esta realidad, la clase social en desventaja sufre directamente sus efectos que se presentan muy interiorizados en una parte muy importante de nuestro alumnado incidiendo, explícita o implícitamente, en las relaciones culturales, emocionales, sociales y ambientales del entorno en que viven (ver Cuadro 1). Se hace necesario de manera urgente una respuesta coherente y real para que se puedan facilitar alternativas positivas al contexto educativo, familiar y social. Desde nuestro punto de vista esta respuesta debe ser, fundamentalmente, educativa, ya que podemos afirmar que la educación y formación ha dejado de ser propiedad exclusiva de la escuela y se ha transformado en una necesidad social y colectiva, para ello las intervenciones sociales son imprescindibles.

Pero estas intervenciones educativas deben cubrir los diferentes aspectos del ser humano y no deben centrarse, exclusivamente, en el aprendizaje de ciertas competencias especializadas. A través de esta exigencia social y colectiva se propone incluir e integrar la transdisciplinaridad en el contexto educativo y, a través de ella, los valores humanos.

\subsection{TRANSDISCIPLINARIDAD Y EDUCACIÓN TRANSDISCIPLINAR}

La transdisciplinaridad es una expectativa parcialmente nueva en el contexto del conocimiento humano. El término surge en Francia en 1970 formulado Jean Piaget, Erich Jantsch y André Lichnerowicz en un seminario internacional sobre los problemas de la enseñanza e investigación en las Universidades.

Ya antes de la formulación del concepto se encuentran posturas epistemológicas que ayudan a una mayor comprensión del mismo. En tal sentido, Ortega y Gasset (1999) nos prevenía de lo que denominó "la barbarie de la especialización", "esa casta de hombres sobremanera extraños que son los especialistas. Son aquellos que saben muy bien su mínimo rincón de universo, pero ignoran de raíz todo el resto." Más recientemente, Morin plantea la situación refiriéndose a la "barbarie en el interior de la ciencia que es el pensamiento disciplinar, la compartimentación en disciplinas." (VALENZUELA, 1992).

La transdisciplinaridad, según Nicolescu (2002), tiene por finalidad el entendimiento del mundo presente del cual uno de los imperativos es la la unidad del conocimiento, su inclinación es la dinámica de la acción inscrita en distintos niveles de la realidad, en la aparición de nuevas lógicas y en la emergencia de la complejidad, convirtiéndose en una perspectiva que complementa la visión que se tiene de la realidad, sin necesidad de negar o excluir otros aspectos como la disciplinaridad, la interdisciplinaridad, la pluridisciplinaridad y la transversalidad, siendo una herramienta en desarrollo que se fundamenta en sistemas complementarios esforzándose en motivar el estudio de la realidad de forma integral. 
Precisamente, la transdisciplinaridad es la forma epistemológica que adopta la teoría de sistemas ya que permite afrontar el conocimiento de forma que se trasciende a las distintas especialidades o disciplinas.

La idea de la transdisciplinaridad no es otra que considerar la realidad como un conjunto de combinaciones que permite una explicación profunda de las complejas realidades del mundo actual (MARTíNEZ, 2007), es decir, podemos especificarla como una expectativa o esperanza que plantea tener en cuenta la realidad como un conjunto de aspectos que permite una explicación más amplia. En este mismso sentido, el informe PISA plantea la necesidad del aprendizaje multidimensional y permanente, de forma que los individuos pueden examinar cuestiones locales, globales e interculturales, comprender y apreciar diferentes perspectivas y visiones del mundo, interactuar con éxito y de manera respetuosa con los demás y actuar de modo responsable hacia la sostenibilidad y el bienestar colectivo (ORGANIZAÇÃO PARA A COOPERAÇÃO E DESENVOLVIMENTO ECONÓMICO, 2018).

A modo de resumen, en el Cuadro 2 se ofece la evolución histórica de las ideas centrales sobre la transdisciplinaridad debidas a sus principales autores. Desde nuestro punto de vista el enfoque transdisciplinar debe priorizarse sobre el clásico enfoque de las disciplinas y, además, es necesario integrar la formación en valores humanos. Esta última es nuestra pequeña aportación al concepto: los valores humanos atraviesan todas las disciplinas y determinan la trascendencia que cada una de ellas tiene para la vida humana. Es fundamental que las cuestiones disciplinares tengan sentido y se juzguen desde sus aportaciones a los valores humanos (el aspecto ético del conocimiento).

Cuadro 2 - Principales ideas entorno a la Transdisciplinaridad

\begin{tabular}{|c|l|l|l|}
\hline Año & \multicolumn{1}{|c|}{ Autor } & \multicolumn{1}{|c|}{ Transdiciplinaridad } & \multicolumn{1}{|c|}{ Observaciones } \\
\hline 1972 & Jantschen & $\begin{array}{l}\text { Reconocimiento de la interdependencia entre todos los } \\
\text { aspectos de la realidad. }\end{array}$ & $\begin{array}{l}\text { Intimidad con la } \\
\text { realidad. }\end{array}$ \\
\hline 1972 & Jack Lee Mahan, Jr & $\begin{array}{l}\text { Necesidad de "reverencia a la vida, al hombre y a la } \\
\text { condición humana". }\end{array}$ & $\begin{array}{l}\text { Respeto, responsabi- } \\
\text { lidad por la vida. }\end{array}$ \\
\hline 1979 & J. J. Kockelmans & $\begin{array}{l}\text { Superar los efectos secundarios negativos de la } \\
\text { especialización para hacer que la educación y la } \\
\text { investigación sea más relevante desde el punto de } \\
\text { vista social. }\end{array}$ & $\begin{array}{l}\text { Abrirse a la objeti- } \\
\text { vidad y subjetividad } \\
\text { social. }\end{array}$ \\
\hline 1994 & $\begin{array}{l}\text { P. Freire } \\
\text { El ser humano, un ser inacabado que busca la } \\
\text { perfección por medio de la educación y se forma con } \\
\text { otros sujetos. }\end{array}$ & $\begin{array}{l}\text { Nadie se educa solo, } \\
\text { las personas se } \\
\text { educan unas con las } \\
\text { otras. }\end{array}$ \\
\hline I Congreso Mundial
\end{tabular}


Educación transdisciplinar...

\begin{tabular}{|l|l|l|l|}
\hline Año & \multicolumn{1}{|c|}{ Autor } & \multicolumn{1}{|c|}{ Transdiciplinaridad } & \multicolumn{1}{c|}{ Observaciones } \\
\hline 1994 & Gibbons et al. & $\begin{array}{l}\text { Colaboración de expertos de diversos campos en } \\
\text { proyectos específicos que trascienden los límites de } \\
\text { sus propias disciplinas. }\end{array}$ & $\begin{array}{l}\text { Cruzar otros hori- } \\
\text { zontes. }\end{array}$ \\
\hline 1996 & Nicolescu & $\begin{array}{l}\text { Se preocupó por el significado del prefijo "trans", ir } \\
\text { más allá de las disciplinas y afirma que se identifica } \\
\text { con un nuevo conocimiento: está entre, a través y } \\
\text { más allá de las disciplinas. }\end{array}$ & $\begin{array}{l}\text { Vínculo íntimo y } \\
\text { profundo con el otro } \\
\text { lado de las fronteras. }\end{array}$ \\
\hline 2008 & Nicolescu & $\begin{array}{l}\text { Enfatiza un conceptolsubjetivo) del mundo desde el } \\
\text { significado de lo vivido. }\end{array}$ & $\begin{array}{l}\text { Historias de vidas } \\
\text { pasadas y presentes. }\end{array}$ \\
\hline McGregor & $\begin{array}{l}\text { La Escuela de Zurich prioriza la interfaz entre ciencia, } \\
\text { sociedad y tecnología en el mundo contemporáneo. }\end{array}$ & $\begin{array}{l}\text { Intercomunicación } \\
\text { científicas y sociales. }\end{array}$ \\
\hline
\end{tabular}

Fuente: sintetizado a partir de Bernstein (2015).

\subsection{UALORES HUMANOS Y EDUCACIÓN EN UALORES}

El ser humano está constituido, entre tantas cosas, por afectividades, cualidades, emociones, sentimientos, razonamiento $y$, de forma inherente a ellas, se desarrollan las acciones que son practicadas individualmente y socialmente. Estas acciones podríamos conectarlas a los valores.

La definición de los valores siempre ha ocupado un espacio importante dentro de la preocupación humana. Cómo se puede especificar y aceptar el significado de lo bueno, bonito, justo y qué determinar como malo, feo, injusto. Estos elementos, decididamente, han desafiado el ser humano a buscar respuestas para guiarse en la vida, a través de motivaciones y decepciones. No obstante, estas respuestas no siempre han sido fáciles (FABELO CORZO, 2004).

La relevancia práctica de los valores y su relación con el ser humano hizo aparecer hacia la segunda mitad del siglo XIX y a lo largo del siglo XX el término axiología, del

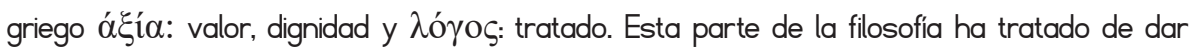
respuestas a cuestiones como la naturaleza de los valores humanos, de dónde surgen y cuál es su fuente. Sin embargo, ante estas cuestiones no se ha podido definir un resultado singular, pues los distintos sistemas ofrecen diferentes interpretaciones y concepciones sobre los valores.

El filósofo argentino Risieri Frondizi propone una explicación sobre los valores que, desde nuestro punto de vista, consigue facilitar una alternativa equitativa y explicativa sobre el concepto. Según este autor, el valor es relacional lo que significa que se trata de una cualidad estructural que surge de la reacción de un sujeto frente a propiedades que se encuentran en un objeto, sin embargo, esa relación no se da en el vacío, sino en una 
situación física y humana determinada (FRONDIZI, 1958). En consecuencia podríamos evidenciar que el valor es complejo y en su estructura íntima actúan elementos objetivos y subjetivos, que se podrían manifestar a través de una visión integradora.

Por otra parte, autores como Emile Durkheim, Lucien Lévy-Brühl y Célestin Bouglé promovieron lo que se ha dado en llamar sociologismo axiológico. Según estos autores es valioso lo que la sociedad califica como tal. La sociedad (relación social), a través de la cultura y de las tradiciones, promoverá y reproducirá los valores. Por lo tanto, estos autores, manifiestan que los valores actúan como entidades objetivas con fuerza imperativa. Eso explica que cada cultura, cada civilización, tendrá los suyos que probablemente serán distintos de los demás (FABELO CORZO, 2004).

Y aún a pesar de esta relatividad social y cultural de los valores, podemos reconocer en la sociedad occidental valores reconocidos que pueden y deben ser fomentados en la enseñanza formal. Como señala Domínguez Chillón (2004),

\footnotetext{
la función de una escuela comprometida con su realidad social, consistirá en posibilitar y potenciar en los niños y niñas el desarrollo de su capacidad crítica, el respeto a la diversidad, la solidaridad, la justicia y la libertad. Ayudará a diagnosticar las realidades sociales, a facilitar los criterios e instrumentos necesarios para entender qué es lo que sucede en la sociedad, a comprender las causas que determinan su naturaleza y evolución, y a buscar soluciones a los problemas que en ella se instalen.
}

Educar en valores no es algo nuevo, ya en la Antigua Grecia se hablaba de educar en las virtudes (ESTEBAN BORA, 2007). En el actual mundo globalizado es necesario seguir formando personas que se integren en la sociedad y participen de la ciudadanía de manera solidaria y responsable (GREVILLA, 1998; GREVILLA; SORIANO, 2000). Sin embargo debemos partir de las experiencias próximas al niño, de lo que conoce, de lo que ha experimentado, es decir, de lo cercano para ir poco a poco llevándolo a lo más lejano (TUTS; MARTíNEZ TENZ, 2006).

En este sentido y dada las condiciones tan especiales de nuestro entorno, hemos decidido que los valores más necesarios son aquellos que afectan y fomentan las relaciones humanas, a saber, el respeto, la responsabilidad y la honestidad. De modo que, a partir de ellos, se puedan derivar otros como la libertad (autonomía), solidaridad, justicia, compromiso con el medio ambiente, etc. 


\section{UALORES Y TRANSDICIPLINARIDAD EN EL MARCO LEGISLATIUO}

Es evidente que no podemos tomar una iniciativa educativa dentro de la educación formal sin tener en cuenta el marco legal en el que integrar nuestra propuesta. Aunque la legislación española sobre el sistema educativo incluye de forma explícita la formación en valores y la interdisciplinaridad, no se incluye específicamente la posibilidad de una aproximación transdisciplinar a la educación en valores. Sin embargo, presenta los suficientes indicios como para incluir nuestra propuesta.

La ley para la mejora de la calidad educativa (LOMCE) (ESPAÑA, 2013) afirma que detrás de "los talentos de las personas están los valores que los vertebran, las actitudes que los impulsan, las competencias que los materializan y los conocimientos que los construyen." Además la ley se basa en valores democráticos básicos y defiende un sistema educativo de calidad, inclusivo, integrador y exigente que, además, garantice la igualdad de oportunidades.

La misma ley pretende proporcionar al alumnado una educación que le permita afianzar su desarrollo personal y su propio bienestar; desarrollar las habilidades sociales, la creatividad y la afectividad; lograr que adquiera los elementos básicos de la cultura, y formarlo para el ejercicio de sus derechos y de sus obligaciones en la vida como ciudadanos. Por ello, los objetivos educativos establecidos (arts. 17 y 23) contribuirán a desarrollar aquellas capacidades relacionadas con el respeto y el desarrollo personal, el respeto a los demás, la mejora de la convivencia, la ciudadanía activa y responsable, el respeto y el cuidado del entorno social, cultural y natural (TUVILLA, 2012).

La LOMCE (ESPAÑA, 2013) mantiene el modelo integrado de convivencia escolar de la LOE (ESPAÑA, 2006), que supone un planteamiento global y sistémico del centro docente, con implicaciones organizativas, recursos específicos para la prevención, el tratamiento y la resolución pacífica y educativa de los conflictos.

Por lo que respecta a la metodología docente, el Decreto n. 11/2016 (ANDALUCÍA, 2016), por el que se establece la ordenación y el currículo de la Educación Secundaria Obligatoria en la Comunidad Autónoma de Andalucía, recoge en el artículo 4 que "se fomentará el enfoque interdisciplinar del aprendizaje por competencias con la realización por parte del alumnado de trabajos de investigación y de actividades integradas que le permitan avanzar hacia los resultados de aprendizaje de más de una competencia al mismo tiempo." Se trata de facilitar la realización de actividades integradas para el desarrollo coordinado de las distintas competencias. Asimismo, el currículo debe incorporar el respeto a las diferencias, incluyendo conexiones con la vida cotidiana y el entorno inmediato del alumnado, así como la necesaria formación artística y cultural. 
$\mathrm{Ni}$ en el decreto citado anteriormente, ni en el resto de normativas a nivel nacional se encuentran referencias a la transdisciplinaridad. Sin embargo, el artículo 10 de este mismo decreto, considera que los centros docentes dentro de su autonomía organizativa y de gestión podrán incluir materias de ampliación de las existentes o una materia de diseño propio. Es precisamente a través de esta flexibilidad donde se introduce la posibilidad de un Proyecto Transdisciplinar en Valores como una asignatura necesaria, con un carácter innovador dentro de la educación secundaria española.

\section{MARCO ESTRATÉGICO/OPERACIONAL: DE LA TEORÍA AL CURRICULUM}

Es indudable la necesidad de conseguir que nuestros sistemas educativos se adapten a los rápidos cambios de las sociedades actuales y al avance del conocimiento, preparando a los educandos para aplicar lo aprendido a la vida real. En la enseñanza obligatoria, además, resulta prioritario que todos los alumnos adquieran el bagaje que necesitan para alcanzar su desarrollo personal e integral, para participar como ciudadanos en la sociedad y para seguir aprendiendo en el futuro.

La adopción de las competencias clave como elemento organizador de la enseñanza a lo largo de la educación básica intenta contribuir a ese objetivo y se ha generalizado en buena parte de los sistemas educativos del mundo en el momento actual (EGIDO GÁLVEZ, 2011). De ahí que en nuestro sistema educativo se incluyan las competencias clave según Orden ECD/65/2015 (ESPAÑA, 2015), por la que se describen las relaciones entre las competencias, los contenidos y los criterios de evaluación de la educación primaria, la educación secundaria obligatoria y el bachillerato.

El trabajo interdisciplinar y transdisciplinar de contenidos a lo largo de las etapas educativas y la relación de los mismos con las competencias básicas, permite cumplir los objetivos de nuestros currículos de manera más satisfactoria, consiguiendo que el alumno sea más partícipe de su propio aprendizaje (KANDIKO, 2012). Este aspecto combinado con la evaluación de sus iguales y con la valoración de lo trabajado tanto individualmente como en el propio grupo asentará más el conocimiento y hará que el alumno sea más consciente de lo que ha aprendido (PLAUBORG, 2009). Una de las dificultades del proyecto es la integración de diferentes formas de concebir el mundo y los saberes, ya que se trata de una población heterogénea. En el proyecto se adopta una visión inclusiva y participativa de forma que cada uno de los grupos culturales conozca y comprenda su propia cultura y la de los otros grupos dentro de la formación curricular exigida por la legislación española. 
En este sentido insertar la transdiciplinaridad en el curriculo implica algunos elementos interesantes que hemos tratado de trasladar a nuestro proyecto:

a) cooperación entre especialistas de diferentes disciplinas. Es necesario que los especialistas colaboren y definan contenidos comunes que se pueden abordar desde diferentes puntos de vista;

b) integración social del conocimiento y de sus repercusiones. El conocimiento y sus prácticas tienen repercusiones socio-ambientales que es necesario comprender y evaluar;

c) integración de visiones multiculturales del mundo. Los propios educandos tienen diferencias culturales (etnias gitana y no gitana) que deben comprender y asumir;

d) adopción de actitudes críticas y diferentes puntos de vista, tanto personales como sociales y culturales.

\section{PROYECTO TRANSDISCIPLINAR EN UALORES}

El PTV surge a través de las observaciones y vivencias adquiridas durante los años 2008-2016 en el entorno social y educativo de Iznalloz. En el análisis socioeducativo (apartado 2.2) se detectan muchas dificultades que afectan a la integración, la convivencia sociocultural, las motivaciones y responsabilidades individuales, los compromisos educativos, los acuerdos sociales y las perspectivas de futuro. Todas ellas se reflejan en actitudes de indiferencia o menosprecio hacia la enseñanza formal, además de producir una fuerte segregación entre la etnia gitana y no gitana mucho más marcada en el entorno social que en el educativo.

Frente a ello, surge la esperanza de trabajar desde el ámbito educativo para realizar acciones dirigidas y coordinadas por medio de la transdisciplinaridad y los valores humanos, ya que en este entorno los educandos están menos segregados. Además, consideramos que los estudiantes de $1^{\circ}$ de la ESO deberían ser el objetivo del proyecto, ya que inician esta etapa educativa y pueden continuar su formación de manera integral hasta concluir la secundaria.

En el Cuadro 3 se sintetizan las líneas maestras fundamentales de proyecto. 
Anfrisia Alessandra Pereira Araujo et al.

Cuadro 3 - Características fundamentales del PTV

\begin{tabular}{|l|l|}
\hline \multicolumn{2}{|c|}{ Proyecto Transdisciplinar en Valores } \\
\hline Participación del educando & Los educandos son sujetos activos y participantes de la acción. \\
\hline Desarrollo del proyecto & Los educadores y los educandos desarrollan el programa conjuntamente. \\
\hline $\begin{array}{l}\text { Implementación del } \\
\text { proyecto }\end{array}$ & $\begin{array}{l}\text { Los educadores elaboran los elementos teóricos y prácticos de forma conjunta } \\
\text { en función del perfil de los educandos. }\end{array}$ \\
\hline Evaluación & Evaluación comprehensiva y continua del desarrollo integral del educando. \\
\hline Vías de comunicación & $\begin{array}{l}\text { Reuniones regulares del equipo docente con trasmisión de información, conoci- } \\
\text { miento y habilidades compartidas. }\end{array}$ \\
\hline Código ético & $\begin{array}{l}\text { El equipo docente se compromete a enseñar, aprender y trabajar en grupo por } \\
\text { medio de acuerdos sociales para implementar el proyecto de manera uniforme. }\end{array}$ \\
\hline Relación con el curriculum & $\begin{array}{l}\text { Los educadores integran sus conocimientos especializados buscando aplicarlos } \\
\text { conjuntamente. }\end{array}$ \\
\hline
\end{tabular}

Fuente: los autores.

\subsection{OBJETIUOS}

El objetivo fundamental del PTV es atravesar y sobrepasar el entorno educativo y llevar al entorno familiar y al entorno social y cultural la importancia de los valores humanos, aunque siempre conectados con una formación teórico-práctica y en relación con la praxis cotidiana de los educandos, teniendo como base un enfoque transdisciplinar.

Dado que la educación en valores es una formación transversal, resulta difíicl adquirirla dentro de las tradicionales disciplinas académicas, ya que estas se suelen centrar en los contenidos especializados. Nuestra propuesta pretende realizar esta formación junto con la formación académica, mediante un proceso de colaboración con los educadores de las asignaturas de $1^{\circ}$ de ESO, tratando de incorporar las estrategias planteadas y la formación en las asignaturas. La estrategia fundamental para conseguir esta integración es facilitar un proceso de adquisición de conocimientos y concienciación que se produzca a través de las actividades del proyecto que se desarrolla oficialmente en el horario lectivo del educando.

Tratamos de motivar a los educandos para desarrollar sus capacidades afectivas, cognitivas, conductuales, emocionales, por medio de contenidos didácticos, basados en valores humanos, emociones básicas, sexualidad humana y ecología. Para ello, se busca favorecer una relación de mutua colaboración y participación entre los educandos.

Este enfoque general se plasma en una serie de objetivos más concretos:

a) conocer las emociones básicas y saber utilizarlas adecuadamente;

b) promover acciones de convivencia y no violencia; 
c) estimular el saber conocer y el saber estar, a través de recursos comunicativos, interpretativos, participativos y desarrollar el razonamiento individual y colectivo;

d) aprender a mejorar las relaciones sociales y desarrollar una consciencia de grupo;

e) desarrollar un pensamiento crítico y reflexivo respecto a la realidad social y ambiental;

f) sensibilizar a las familias y a la comunidad sobre la importancia social de la formación en valores de estos educandos.

\subsection{CONTENIDOS}

Como se trata de un proyecto pionero y novedoso, los materiales didácticos previamente elaborados son escasos, por ello, hemos tenido que generarlos partiendo de los contenidos de las otras asignaturas, realizando una adaptación de los mismos a nuestra propuesta de trabajo. De esta forma hemos logrado la inclusión de temas de las demás asignaturas en el proyecto, abordando el trabajo con valores desde diferentes perspectivas de las ciencias naturales, exactas y sociales. Al inicio del curso se reparten entre los educandos cuadernos didácticos que son recogidos al final de cada trimestre. A su vez, el material se ha presentado a través de diapositivas y vídeos educativos. Además, cada educando dispone de un cuaderno personal en el que toma notas de las cuestiones planteadas en las clases. Los contenidos educativos han sido divididos como se muestra en el Cuadro 4.

Cuadro 4 - Contenidos PTV

\begin{tabular}{|c|c|c|}
\hline $\begin{array}{c}\text { Primer trimestre } \\
\text { (septiembre- diciembre }\end{array}$ & $\begin{array}{l}\text { Segundo trimestre } \\
\text { (enero-marzo) }\end{array}$ & $\begin{array}{l}\text { Tercer trimestre } \\
\text { (marzo-junio) }\end{array}$ \\
\hline Los Valores Humanos & Emociones Básicas & Sexualidad humana y ecología \\
\hline $\begin{array}{l}\text { - Definiciones y casos } \\
\text { prácticos incluyendo los } \\
\text { valores: respeto, responsa- } \\
\text { bilidad y honestidad } \\
\text { - Visualización e interpreta- } \\
\text { ción de documentales, peli- } \\
\text { culas y videos educativos }\end{array}$ & $\begin{array}{l}\text { - Constitución del ser } \\
\text { humano y las emociones } \\
\text { básicas } \\
\text { - Factores que influyen las } \\
\text { emociones básicas. } \\
\text { - Análisis e identificación de } \\
\text { las emociones individuales y } \\
\text { colectivas. }\end{array}$ & $\begin{array}{l}\text { - Conceptos, definiciones y opiniones: Sexo, } \\
\text { identidad de género, rol de género, orientación } \\
\text { sexualidad. } \\
\text { - Relación del cuerpo con el entorno social y } \\
\text { ambiental. } \\
\text { - Evolución y origen humanos. } \\
\text { - La fotosíntesis y los gases de efecto invernadero. } \\
\text { - Significado de los elementos naturales para a } \\
\text { vida humana. } \\
\text { - Campañas de reciclado. }\end{array}$ \\
\hline
\end{tabular}

\footnotetext{
Fuente: los autores.
} 


\subsection{METODOLOGÍA Y ACTIUIDADES}

La metodología de enseñanza y aprendizaje está basada en los modelos integradores (GONZÁLEZ et al., 1999) que añaden tanto aspectos objetivistas (cognitivos) como subjetivistas (motivacionales y volitivos) y que buscan que las personas se encuentren, a través de la racionalidad, la autonomía y la comunicación. Esta metodología se basa en acciones conscientes a través de los métodos colaborativo, comunicativo y participativo, que impulsan la implicación de los educandos, los educadores, la familia y el entorno social. Se trata de favorecer una relación de empatía, de ayuda mutua y confianza, de modo que todos y todas colaboren en el desarrollo del proyecto.

Para cubrir los objetivos anteriores y desarrollar la metodología, se propone la realización de actividades y temas que estén conectadas con los objetivos y que además se conecten con las disciplinas académicas, tanto científicas como sociales y humanas.

Las actividades están centradas y dirigidas a los educandos y persiguen la participación y el trabajo en grupo, con la finalidad de lograr una relación de confianza y respeto mutuo, favoreciendo un entorno cálido en el que se sientan seguros para hablar con naturalidad sobre sus experiencias, relaciones, problemas, en un ambiente participativo y común que será el espacio grupal. Este espacio facilitará la posibilidad de un trabajo socio-educativo. Todo ello, a través de un aprendizaje significativo por medio de las prácticas didácticas, utilizando un análisis reflexivo: pensar, indagar, aprender, dudar, conocer, motivarse, decidir e integrarse.

Para ello se propuso intercalar esta formación en valores dentro del programa académico, como hemos explicado en los objetivos. La frecuencia de estas clases ha sido de una hora a la semana para cada grupo y hemos contado con la colaboración y participación de los Departamentos de Biología y Geología, Departamento de Geografía e Historia y Departamento de Música.

Las actividades realizadas han estado basadas en estrategias educativas características de la educación en valores. El Cuadro 5 muestra algunos ejemplos de ellas. 
Cuadro 5 - Ejemplos de actividades educativas

\begin{tabular}{|c|c|c|c|}
\hline $\begin{array}{c}\text { ¿̇Qué son los valores y } \\
\text { para que sirven? }\end{array}$ & $\begin{array}{c}\text { Descubriendo las emocio- } \\
\text { nes básicas. }\end{array}$ & $\begin{array}{l}\text { Rompiendo tabúes sobre } \\
\text { la sexualidad humana. }\end{array}$ & $\begin{array}{l}\text { La Naturaleza antes que } \\
\text { cualquier otro tema. }\end{array}$ \\
\hline $\begin{array}{l}\text { - Cómo los valores nos } \\
\text { guían en las actividades } \\
\text { vitales. } \\
\text { - Estudio de casos } \\
\text { prácticos. } \\
\text { - Análisis crítico de docu- } \\
\text { mentales. }\end{array}$ & $\begin{array}{l}\text { - Cómo los factores } \\
\text { biológicos, culturales, físi- } \\
\text { cos, sociales, racionales, } \\
\text { religiosos, etc. influyen } \\
\text { en las emociones del ser } \\
\text { humano. } \\
\text { - Elaboración y presen- } \\
\text { tación del emocionario de } \\
\text { grupo }\end{array}$ & $\begin{array}{l}\text { - Cuerpo asexuado y } \\
\text { sexuado. } \\
\text { - Deferencias entre } \\
\text { cuerpo humano y las } \\
\text { máquinas. } \\
\text { - Interpretar e identificar } \\
\text { la sexualidad a través de } \\
\text { narraciones. }\end{array}$ & $\begin{array}{l}\text { - Planeta Tierra y } \\
\text { prácticas de un buen } \\
\text { ciudadano. } \\
\text { - Reciclado de aparatos } \\
\text { electrónicos usados. } \\
\text { - Reforestación y medio } \\
\text { ambiente. }\end{array}$ \\
\hline
\end{tabular}

Fuente: los autores.

\subsection{EUALUACIÓN DE LOS EDUCANDOS}

En todo trabajo socio-educativo evaluar es una labor importante y fundamental para saber si se está cumpliendo con los objetivos planteados y la metodología propuesta. Por tanto, cuanto más información se recoja y se observen, la evaluación será más exitosa y nos ayudará en actividades futuras.

Nuestro proceso de evaluación ha sido realizado de manera sistemática, teniendo en cuenta el proceso evolutivo del educando, tanto dentro del aula como fuera de ella. Además, la evaluación ha tenido un carácter continuo, global e integral (PÉREZ, 2006), teniendo en cuenta todas las actividades realizadas, las actitudes del educando, los resultados de los trabajos, cuidado y creatividad con los materiales didácticos, las infraestructuras y el entorno ambiental.

La comunicación y las decisiones de la evaluación han sido tomadas en reuniones del equipo docente con dos propósitos fundamentales:

a) proporcionar retroalimentación descriptiva e informativa de forma oportuna a todos los involucrados en el proyecto, permitiendo identificar los logros y dificultades con respecto a los objetivos del proyecto, para mantener las fortalezas y oportunidades y combatir las debilidades y amenazas;

b) proporcionar a los educadores, madres/padres, equipo directivo, administraciones locales, una valoración global sobre el nivel en que los estudiantes alcanzaron los objetivos propuestos en el proyecto. 
El Cuadro 6 resume las herramientas, los procedimientos y los criterios de calificación utilizados en la evaluación.

Cuadro 6 - Herramientas, procedimientos y criterios de calificación

\begin{tabular}{|l|l|l|}
\hline \multicolumn{1}{|c|}{ Procedimientos } & \multicolumn{1}{|c|}{ Instrumentos } & \multicolumn{1}{c|}{ Criterios de calificación } \\
\hline - Observación en el aula y fuera & - Listas de observación. & - Los contenidos: tener las tareas \\
de ella. & - Cuaderno y material didáctico del & y anotaciones realizadas. \\
- Análisis y corrección del cua- & educando. & - Nivel consecución de tareas. \\
derno de PTV realizadas por el & - Registro anecdótico. & - Expresión escrita, crítica y \\
educando. & - Diario de campo y observaciones. & reflexiva. \\
- Percepción del cuadernillo didácti- & - Ejercicios escritos y prácticos. & - Creatividad y responsabilidad con \\
co (cuidado y creatividad). & - Rúbricas de productos finales. & el cuadernillo didáctico lorden y \\
- Colaboración y rectificación de & & limpieza). \\
tareas orales lexposiciones orales, & & - Interés, esfuerzo, asistencia y \\
escritas y audiovisuales. & puntualidad. \\
- Autoevaluación y coevaluación & & - Actitudes dentro y fuera del aula. \\
del educando. & & \\
\hline
\end{tabular}

Fuente: los autores.

\subsection{EUALUACIÓN DEL PROYECTO Y SUS RESULTADOS}

A través de la evaluación se pretendió detectar las potencialidades y dificultades de todo el proceso de enseñanza y aprendizaje desarrollado en PTV. La Tabla 1 muestra cómo han mejorado los resultados de la evaluación a lo largo del curso en términos de alumnado que superó positivamente el PTV. Hemos podido concluir que la adecuación de los elementos de la programación a las características de los educandos ha sido adecuada, según:

\footnotetext{
a) el nivel de interacción entre los educandos, educadores y comunidad educativa;

b) los trabajos en grupo han favorecido la integración y participación consolidado un clima eficaz de contraste de opiniones;

c) además, ha mejorado considerablemente la participación de las madres y padres en las actividades prácticas de sus hijos, aunque se echa en falta una mayor implicación de la etnia gitana.
}

Tabla 1 - Resultados positivos del PTV en el curso 2017/2018 
Educación transdisciplinar...

\begin{tabular}{|c|c|c|c|c|}
\hline Grupo & $\mathrm{N}^{\circ}$ Educandos & $1^{\circ}$ Trimestre & $2^{\circ}$ Trimestre & $3^{\circ}$ Trimestre \\
\hline $1^{\circ} \mathrm{A}$ & 23 & $46 \%$ & $57 \%$ & $97 \%$ \\
\hline $1^{\circ} \mathrm{B}$ & 23 & $47 \%$ & $56 \%$ & $94 \%$ \\
\hline $1^{\circ} \mathrm{C}$ & 22 & $63 \%$ & $60 \%$ & $99 \%$ \\
\hline $1^{\circ} \mathrm{D}$ & 26 & $57 \%$ & $84 \%$ & $95 \%$ \\
\hline Total & 94 & & & \\
\hline
\end{tabular}

Fuente: los autores.

En nuestra programación se ha presentado a los educandos la realización de campañas concretas de reciclado y movilización ciudadana para fomentar el respeto al medio ambiente, la responsabilidad y la colaboración en la conservación del Planeta. Hemos conseguido, además, que los educandos empaticen con problemáticas muy distantes como la explotación de los recursos naturales o la desaparición de especies animales. Además, es importante resaltar que por medio de estas campañas y del contenido del PTV hemos logrado, mediante acciones ciudadanas y encuentros con autoridades locales que se aprueben medidas políticas de intervención medioambiental que afectan directamente a la población, como por ejemplo, la prohibición expresa de circos con animales o la recogida de dispositivos electrónicos.

Como afirma Cardonna (2014), la noción de sostenibilidad ha evolucionado de un concepto a un movimiento que involucra no solo a la ciencia, el gobierno y la industria, sino también la participación ciudadana. En este sentido es importante trasladar los contenidos estudiados y aprendidos en el aula al entorno social y, a la vez, solicitar la cooperación y la responsabilidad de la comunidad para construir un ambiente armonioso, fructífero y con perspectivas de futuro.

\section{CONSIDERACIONES FINALES}

La educación posibilita al joven reanudar su potencial, desarrollar sus habilidades, y asegurar competencias adquiridas. Una educación transdisciplinar ofrece una perspectiva de cambio al proporcionar una integración de conocimientos y experiencias que son necesarios en la sociedad actual para formar personas que sean capaces de intervenir en entornos cada vez más complejos. Por otra parte, educar en valores en el contexto de disciplinas más cognitivas, proporciona una visión integral de los sujetos educativos, disminuyendo la fragmentación del conocimiento y su adaptación al ser humano.

Nuestro Proyecto Transdisciplinar en Valores ha permitido abrir la educación a la vida cotidiana y a la vida práctica, y ha favorecido una convivencia colectiva y participativa. Además, ha facilitado que el educando asimile los contenidos de las diferentes asignaturas 
de una manera enlazada, continua e inclusiva visualizando así su importancia y su conexión con su realidad diaria y vital. Estos resultados, deseables en entornos socialmente estables, son especialmente importantes en entornos desfavorecidos dónde la población se encuentra en riesgo de exclusión social. Cómo en nuestro caso, comprender los acontecimientos que ocurren alrededor permite integrar en la sociedad a aquellos grupos de población en riesgo de exclusión social, al hacer que se comprometan entre sí, con el medio ambiente y con la realidad en la que habitan.

\section{REFERENCIAS}

ANDALUCÍA. Decreto n. 111, de 14 de junio de 2016. Establece la ordenación y el currículo de la Educación Secundaria Obligatoria en la Comunidad Autónoma de Andalucía. BOJA: Boletín Oficial de la Junta de Andalucía n. 122, p. 27, 14 jun. 2016. Disponible en: http://www.juntadeandalucia.es/boja/2016/122/ B0JA16-122-00223.pdf. Acceso en: 28 jun. 2018.

BENDIT, R.; HAHN-BLEIBTREU, M. La Transición a la Vida Activa. Revista de Educación, Madrid, n. 351, sept./dic. 2008.

BERNSTEIN, J. H. Transdisciplinarity: A review of its origins, development, and current issues. Journal of Research Practice, New York: Retrieved, v. 11, n. 1, 2015. Disponible en: http://jrp.icaap.org/index.php/ jrp/article/view/510/412. Acceso en: 29 mayo 2018.

BORDIEU, P. El Sentido Práctico. 1. ed. Buenos Aires: Siglo XXI, 2007.

CARDONNA, J. L. Sustainaty: A history. Oxford, Reino Unido: Oxford University Press, 2014.

DOMÍNGUEZ CHILLÓN, G. Los valores en la Educación Infantil. Madrid: Editorial La Muralla, 2004.

EGIDO GÁLVEZ, I. Las competencias clave como elemento central del currículo de la enseñanza obligatoria: un repaso a las experiencias europeas. Revista Española de Educación Comparada, Madrid: UNED, n. 17, p. 239-262, 2011.

ESPAÑA. Ley n. 2, de 3 de junio de 2006. Ley Orgánica de Educación. BOE: Boletín Oficial del Estado, Madrid, 3 jun. 2006. Disponible en: https://www.boe.es/buscar/pdf/2006/BOE-A-2006-7899-consolidado.pdf. Acceso en: 20 mayo 2018.

ESPAÑA. Ley n. 8, de 9 de diciembre de 2013. Ley Orgánica para la Mejora de la Calidad Educativa. BOE: Boletín Oficial del Estado, Madrid, 9 dic. 2013. Disponible en: https://www.educastur.es/-/lomce-ley-organica-para-la-mejora-de-la-calidad-educativa Acceso en: 20 mayo 2018.

ESPAÑA. Orden ECD/65, de 21 de enero de 2015. BOE: Boletín Oficial del Estado, Madrid, 21 enero 2015. Disponible en: https://boe.es/buscar/pdf/2015/B0E-A-2015-738-consolidado.pdf. Acceso en: 20 mayo 2018. 
ESTEBAN BORA, F. Lluvia de valores. Barcelona: Ediciones Ceac, 2007.

FABELO CORZO, J. R. Los valores y sus desafios actuales. Libro en red. 2004. Disponible en: http:// www.insumisos.com/lecturasinsumisas/Los\%20valores\%20y\%20los\%20desafios\%20actuales.pdf. Acceso en: 6 jun. 2018.

FORO CIUDAD. Evolución de la población. Demografia de Iznnalloz, 2017. Disponible en: https://www. foro-ciudad.com/granada/iznalloz/habitantes.html. Acceso en: 6 jun. 2018.

FRONDIZI, R. żQué son los valores? 1. ed. México: Breviarios del Fondo de Cultura Económica, 1958.

GONZÁLEZ, R. et al. Un modelo integrador explicativo de las relaciones entre metas académicas. Revista de Investigación Educativa, La Coruña, v. 17, n. 1, p. 47-70, 1999.

GREVILLA, E. Educación y Valores. Revista Española de Pedagogía, España, v. 1, n. 84, 1998.

GREVILLA, E.; SORIANO, A. La Educación Hoy. Conceptos, interrogantes y valores. Granada: Grupo Editorial Universitario, 2000.

INSTITUTO NACIONAL DE ESTADISTICA. Estructura Demográfica. 2017. Disponible en: http://www.ine.es/. Acceso en: 6 jun. 2018.

KANDIKO, C. B. Leadership and creativity in higher education: The role of interdisciplinarity. London Review of Education, v. 10, n. 2, p. 191-200, 2012.

LEÉGEOIS, J. P. Minoría y escolaridad: el paradigma gitano. Madrid: Presencia Gitana, 1998.

MARTÍNEZ, M. Conceptualización de la transdisciplinariedad. Polis, v. 16, 2007. Disponible en: http://polis. revues.org/4623. Acceso en: 30 mayo 2018.

NICOLESCU, B. Manifesto of transdiciplinarity. [S. ll: Suny Press, 2002.

ORTEGA Y GASSET, J. La rebelión de las masas (1929). Madrid: S.L.U. Espasa Libros, 1999.

ORGANIZAÇÃO PARA A COOPERAÇÃO E DESENVOLVIMENTO ECONÓMICO. Marco de Competencia Global. Estudio PISA. Preparar a nuestros jóvenes para un mundo inclusivo y sostenible. [S. l.: Ministerio de Educación, Cultura y Deporte, 2018.

PEREIRA, A. A. Diagnóstico de Intervención Socioeducativa. 2016. Máster - Universidad de Granada, Granada, 2016.

PÉREZ, R. Evaluación de Programas Educativos. Madrid: La Muralla, 2006. 
PLAUBORG, H. Opportunities and limitations for learning within teachers' collaboration in teams: perspectives from action learning. Action learning; Research and Practice, v. 6, n. 1, p. 25-34, 2009.

SISTEMA de Información Multiterritorial de Andalucía (SIMA). Instituto de Estadística y Cartografía de Andalucía, 2017. Disponible en: https://www.juntadeandalucia.es/institutodeestadisticaycartografia/ iea/consultasActividad.jsp?Cod0per=104\&sub=38120. Acceso en: 5 jun. 2018.

TUTS, M.; MARTíNEZ TEN, L. Educación en valores y ciudadanía. Madrid: La Catarata, 2006.

TUVILLA, J. La educación en valores y la LOMCE. Eurydice, España, 2012. Disponible en: http://josetuvillarayo.es/files/06_LOMCE_Valores_-definitivo.pdf. Acceso: 25 mayo 2018.

VALENZUELA, J. Babelia. [Entrevista cedida a] Edgar Morin. El País, Madrid, १८ jul. 1992.

Direcciones para correspondencia: Calle del Puente, १7, १८५50, Iznalloz, Granada, España; anfrisiaa@ gmail.com 